and Sylvius de Le Boe based their practice upon astrology and alchemy. Borelly and Boerhaave applied the laws of mechanics to medical theories. Stall explained psychical phenomena by the illimitable power of the soul superimposed upon the body, and acting as a conservative sentinel upon the corporeal manifestation. Cullen explained everything upon an exercise of the nervous force. Gaubo and Selle adopted the humoral patholory. Brown reduced all infirmities to the orders sthenic and asthenic; his notions were drawn from the ancient theory of Themison, who ascribed every deviation to constriction and laxation. The illustrious Bartheus originated the idea of the vital power. Broussais charged all maladies to irritation of the solids. Rasori founded in Italy the school of counter-stimulation, and exaggerated the necessity of excessive medication, in striking contrast with the system of Hahnemann and the conceptions of homœopathia."
Attleboro', Nov. 24, 1852.
[To be continued.]
E. SANFord, M.D.

\title{
SINGULAR CAUSE OF DEATH.
}

[Uommunicated for the Boston Medical and Surgicnl Jnurnal.]

Tre following singular and fatal termination of life in the case of Dr. Charles C. Sheppard, from an inoculated virus, may not be unacceptable or uninstructive to the readers of the Boston Medical and Surgical Journal.

Dr. S. was a young gentleman of steady babits, and of peculiarly mild and aniable characteristics in all his thoughts and actions. His temperament was the sero-lymphatic. Physically he was tall in stature, of "spare habit," and apparently weak in his limbs. He recently took his medical degree in one of our first medical colleges, and immediately afterwards commenced practising medicine in our sister city Hoboken, N. J. As "a beginner," his practice was, as usual, limited to the humbler classes of society. He was "called in" to attend a case of midwifery, and during the parturition of his patient, his hands of course were moistened with the secretions attending this effort of nature. With them he rubbed his lower lip, which happened to be affected with a fissure in its centre, arising from a cold, dryness, or some other cause. Immediately after doing so, his lip commenced swelling, superinduced, it is presumed, from some peculiar virus contained in the secretions-for the woman apparently enjoyed good health, and recovered as women usually do under such circumstances. The swelling from the lip gradually spread, until it had implicated the whole of his face and scalp with a sort of erysipelatous phlegmonous inflammation, which terminated his life in a few hours.

A similar accident occurred in Philadelphia two years since, which fortunately did not terminate in death, but deprived the physician of the use of his arm upwards of a year, fears being entertained that amputation would be necessary. The physician had inoculated a recent wound made by an axe, on bis dexter finger. 
Dr. Charles C. Sheppard, unknown to the medical profession in life, by his unfortunate death and its singular cause, may perchance be the means of proving a beneficial warning to medical men of the dangers attending carelessness, or rather the want of precaution, in obstetric practice.

New York, Dec. 18, 1852.

A. C. Castle, M.D.

\section{POISONING BY TOBACCO.}

To the Editor of the Boston Medical and Surgical Journal.

Sir,-I transmit to you a summary report of a case of poisoning from the use of tobacco, as I copy it from my note-book, which you are at liberty to dispose of as you think proper.

October 6th, 9 o'clock, P.M.-Was called, in haste, to visit the patient, a male child, æt. 7 days, previously healthy. Learned the following particulars. There had been given to the child, at about 8 o'clock, P.M., for the purpose of inducing quietness through the night, about two tablespoonsful of water impregnated with tobacco smoke (the smoke blown through a new pipe into the water, until the operator bad become nauseated; and was strong enough to make the tongue of the mother "smart dreadfully," when tasted). The patient presented the following appearances. Entire system flaccid, pallid, eyes closed, comatose ; pulse not perceptible at wrist, and action of heart scarcely so in præcordial region; respiration spasmodic, deep, six per minute; deglutition difficult (impossible, except when the article to be swallowed was placed low in the throat); temperature high.

Treatment.-Gave a teaspoonful of wine; applied tr. camph. and aqua ammonia externally, but without any immediate apparent effect. In five minutes administered carb. amm., grs. v., in solution. This seemed to arouse the patient for a few minutes, and he breathed easier and oftener. Action of heart increased. Gave stimulating enema ; and externally, frictions with stimulants.

9.1 o'clock.-Gradually relapsed. Respiration and action of heart ceased ; comatose. Treatment.-Transition baths; artificial respiration. Soon revived, so as to respire with a gasp. Heart acts. Carb. amm., grs. iij.

12 o'clock.-Was kindly assisted by my friend, Dr. S. Tuttle. Administered ether sulph. by inhalation. No material change.

Suffice it to say, that from 9 o'clock, P.M., of the 6th, until 4 o'clock, A.M. of the 7th (eight hours from the time he took the tobacco-water), the treatment pursued was stimulants externally and internally, with baths, \&c., pro re nata, and artificial respiration (at least five hours of the time), when the patient, after having respired himself for about thirty minutes, suddenly expired, and no effort which we could bring to bear would arouse him. Electricity, and the nitrous-oxide water of Dr. Ziegler, might possibly have done it ; but unfortunately, I had neither of them at my command at the moment.

Autopsy. Oct. 8, 11 o'clock, A.M.-Present, Drs. Hoskins and Weaks. Ext. Appearances.-An unusual redness over the entire surface. 